\title{
Biomarkers for depression: recent insights, current challenges and future prospects
}

\author{
This article was published in the following Dove Press journal: \\ Neuropsychiatric Disease and Treatment \\ 10 May 2017 \\ Number of times this article has been viewed
}

\author{
Rebecca Strawbridge' \\ Allan $\mathrm{H}$ Young ${ }^{1,2}$ \\ Anthony J Cleare ${ }^{1,2}$ \\ 'Centre for Affective Disorders, \\ Department of Psychological \\ Medicine, Institute of Psychiatry, \\ Psychology and Neuroscience, King's \\ College London, ${ }^{2}$ South London and \\ Maudsley NHS Foundation Trust, \\ London, UK
}

\begin{abstract}
A plethora of research has implicated hundreds of putative biomarkers for depression, but has not yet fully elucidated their roles in depressive illness or established what is abnormal in which patients and how biologic information can be used to enhance diagnosis, treatment and prognosis. This lack of progress is partially due to the nature and heterogeneity of depression, in conjunction with methodological heterogeneity within the research literature and the large array of biomarkers with potential, the expression of which often varies according to many factors. We review the available literature, which indicates that markers involved in inflammatory, neurotrophic and metabolic processes, as well as neurotransmitter and neuroendocrine system components, represent highly promising candidates. These may be measured through genetic and epigenetic, transcriptomic and proteomic, metabolomic and neuroimaging assessments. The use of novel approaches and systematic research programs is now required to determine whether, and which, biomarkers can be used to predict response to treatment, stratify patients to specific treatments and develop targets for new interventions. We conclude that there is much promise for reducing the burden of depression through further developing and expanding these research avenues.
\end{abstract}

Keywords: mood disorder, major depressive disorder, inflammation, treatment response, stratification, personalized medicine

\section{Introduction Challenges in mental health and mood disorders}

Although psychiatry has a disease-related burden greater than any single other medical diagnostic category, ${ }^{1}$ a disparity of esteem is still apparent between physical and mental health across many domains including research funding ${ }^{2}$ and publication. ${ }^{3}$ Among the difficulties that mental health faces is a lack of consensus surrounding classification, diagnosis and treatment that stems from an incomplete understanding of the processes underlying these disorders. This is highly apparent in mood disorders, the category which comprises the single largest burden in mental health. ${ }^{3}$ The most prevalent mood disorder, major depressive disorder (MDD), is a complex, heterogeneous illness in which up to $60 \%$ of patients may experience some degree of treatment resistance that prolongs and worsens episodes. ${ }^{4}$ For mood disorders, and in the broader field of mental health, treatment outcomes would likely be improved by the discovery of robust, homogeneous subtypes within (and across) diagnostic categories, by which treatments could be stratified. In recognition of this, global initiatives to delineate functional subtypes are now in progress, such as the research domain criteria. ${ }^{5}$ It has been posited that biologic markers are priority candidates for subtyping mental disorders. ${ }^{6}$ 


\section{Improving response to treatments for depression}

Despite an extensive range of treatment options for major depression, only approximately a third of patients with MDD achieve remission even when receiving optimal antidepressant treatment according to consensus guidelines and using measurement-based care, and rates of treatment response appear to fall with each new treatment. ${ }^{7}$ Furthermore, treatment-resistant depression (TRD) is associated with increased functional impairment, mortality, morbidity and recurrent or chronic episodes in the long term. ${ }^{8,9}$ Thus, obtaining improvements in treatment response at any clinical stage would afford wider benefits for overall outcomes in depression. Despite the substantial burden attributable to TRD, research in this area has been sparse. Definitions of TRD are not standardized, in spite of previous attempts: ${ }^{4}$ some criteria require only one treatment trial that fails to achieve a 50\% symptom score reduction (from a validated measure of depression severity), while others require nonachievement of full remission or nonresponse to at least two adequately trialed antidepressants of different classes within an episode to be considered TRD., ${ }^{4,10}$ Furthermore, the staging and prediction of treatment resistance is improved by adding the key clinical features of severity and chronicity to the number of failed treatments. ${ }^{9,11}$ Nevertheless, this inconsistency in definition renders interpreting the research literature on TRD an even more complex task.

In order to improve response to treatments, it is clearly helpful to identify predictive risk factors of nonresponse. Some general predictors of TRD have been characterized, including a lack of full remission after previous episodes, comorbid anxiety, suicidality and early onset of depression, as well as personality (particularly low extraversion, low reward dependence and high neuroticism) and genetic factors. ${ }^{12}$ These findings are corroborated by reviews synthesizing the evidence separately for pharmacologic ${ }^{13}$ and psychological $^{14}$ treatment for depression. Antidepressants and cognitivebehavioral therapies show approximately comparable efficacy, ${ }^{15}$ but due to their differing mechanisms of action might be expected to have different predictors of response. While early-life trauma has long been associated with poorer clinical outcomes and reduced responses to treatment, ${ }^{16}$ early indications suggest that people with a history of childhood trauma might respond better to psychological than pharmacologic therapies. ${ }^{17}$ Despite this, uncertainty prevails and little personalization or stratification of treatment has reached clinical practice. ${ }^{18}$
This review focuses on the evidence supporting the utility of biomarkers as potentially useful clinical tools to enhance treatment response for depression.

\section{Biomarkers: systems and sources}

Biomarkers provide a potential target for identifying predictors of response to various interventions..$^{19}$ The evidence to date suggests that markers reflecting the activity of inflammatory, neurotransmitter, neurotrophic, neuroendocrine and metabolic systems may be able to predict mental and physical health outcomes in currently depressed individuals, but there is much inconsistency between findings. ${ }^{20}$ In this review, we focus on these five biologic systems.

To attain a full understanding of molecular pathways and their contribution in psychiatric disorders, it is now considered important to assess multiple biologic "levels", in what is popularly referred to as an "omics" approach. ${ }^{21}$ Figure 1 provides a depiction of the different biologic levels at which each of the five systems can be assessed, and the potential sources of markers on which these assessments can be undertaken. However, note that while each system can be inspected at each omics level, the optimal sources of measurement clearly vary at each level. For example, neuroimaging provides a platform for indirect assessment of brain structure or function, while protein examinations in blood directly assess markers. Transcriptomics ${ }^{22}$ and metabolomics ${ }^{23}$ are increasingly popular, offering assessment of potentially huge numbers of markers, and the Human Microbiome Project is now attempting to identify all microorganisms and their genetic composition within humans. ${ }^{24}$ Novel technologies are enhancing our ability to measure these, including through additional sources; for example, hormones such as cortisol can now be assayed in hair or fingernails (providing a chronic indication) or sweat (providing a continuous measurement), ${ }^{25}$ as well as in blood, cerebrospinal fluid, urine and saliva.

Given the number of putative sources, levels and systems involved in depression, it is not surprising that the scale of biomarkers with translational potential is extensive. Particularly, when interactions between markers are considered, it is perhaps unlikely that examining single biomarkers in isolation will yield findings fruitful for improving clinical practice. Schmidt et $\mathrm{al}^{26}$ proposed the use of biomarker panels and, subsequently, Brand et $\mathrm{al}^{27}$ outlined a draft panel based on prior clinical and preclinical evidence for MDD, identifying 16 "strong" biomarker targets, each of which is rarely a single marker. They comprise reduced gray matter volume (in hippocampal, prefrontal cortex and basal ganglia regions), circadian cycle changes, hypercortisolism and other 


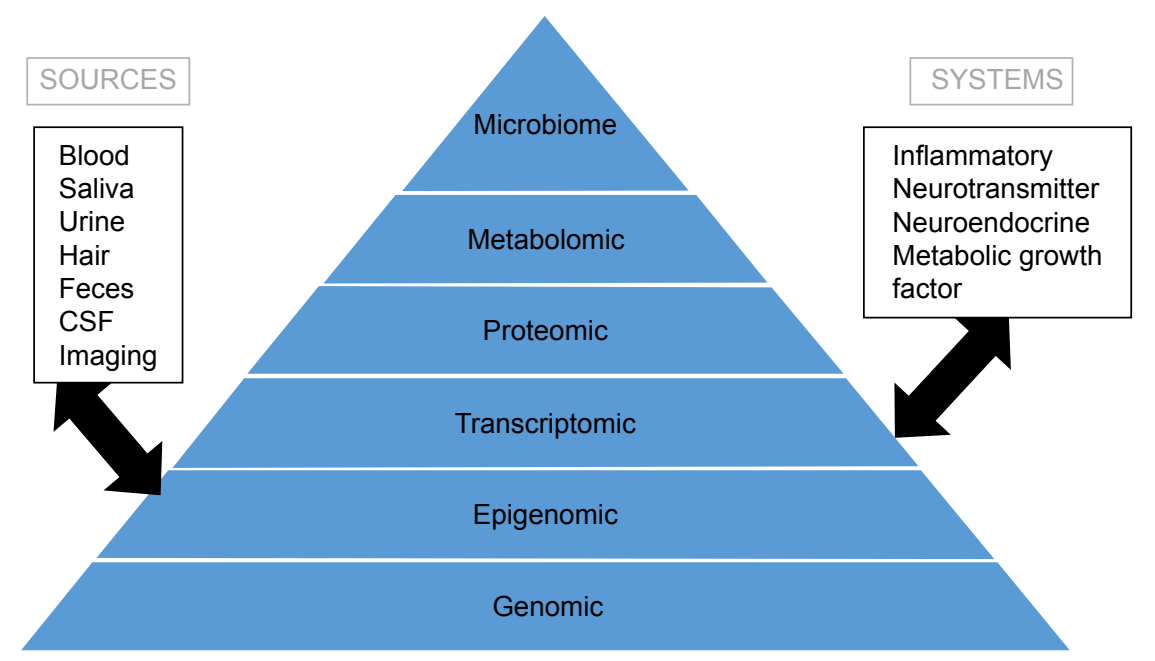

Figure I Potential biomarkers for depression: biological levels, sources and systems.

Notes: See also the study by Suravajhala et $\mathrm{a}^{2{ }^{21}}$ for further definitions and discussion around the genome (all genetic material in an organism), epigenome (all changes to genetic material), transcriptome (all RNA transcripts from genetics), proteome (all proteins expressed in an organism), metabolome (all small-molecule chemicals in an organism) and microbiome (all genes of microbes in an organism).

Abbreviation: CSF, cerebrospinal fluid.

representations of hypothalamic-pituitary-adrenal (HPA) axis hyperactivation, thyroid dysfunction, reduced dopamine, noradrenaline or 5-hydroxyindoleacetic acid, increased glutamate, increased superoxide dismutase and lipid peroxidation, attenuated cyclic adenosine $3^{\prime}, 5^{\prime}$-monophosphate and mitogen-activated protein kinase pathway activity, increased proinflammatory cytokines, alterations to tryptophan, kynurenine, insulin and specific genetic polymorphisms. These markers have not been agreed by consensus and could be measured in various ways; it is clear that focused and systematic work must address this enormous task in order to prove their clinical benefits.

\section{Aims of this review}

As a deliberately broad review, this article seeks to determine the overall needs for biomarker research in depression and the extent to which biomarkers hold real translational potential for enhancing response to treatments. We begin by discussing the most important and exciting findings in this field and direct the reader to more specific reviews pertaining to relevant markers and comparisons. We outline the current challenges faced in light of the evidence, in combination with needs for reducing the burden of depression. Finally, we look ahead to the important research pathways for meeting current challenges and their implications for clinical practice.

\section{Recent insights}

The search for clinically useful biomarkers for people with depression has generated extensive investigation over the last half a century. The most commonly used treatments were conceived from the monoamine theory of depression; subsequently, neuroendocrine hypotheses gained much attention. In more recent years, the most prolific research has surrounded the inflammatory hypothesis of depression. However, a large number of relevant review articles have focused across all five systems; see Table 1 and below for a collection of recent insights across biomarker systems. While measured at many levels, blood-derived proteins have been examined most widely and provide a source of biomarker that is convenient, cost-effective and may be closer to translational potential than other sources; thus, more detail is given to biomarkers circulating in blood.

In a recent systematic review, Jani et $\mathrm{al}^{20}$ examined peripheral blood-based biomarkers for depression in association with treatment outcomes. Of only 14 studies included (searched up until early 2013), 36 biomarkers were studied of which 12 were significant predictors of mental or physical response indices in at least one investigation. Those identified as potentially representing risk factors for nonresponse included inflammatory proteins: low interleukin (IL)-12p70, ratio of lymphocyte to monocyte count; neuroendocrine markers (dexamethasone nonsuppression of cortisol, high circulating cortisol, reduced thyroid-stimulating hormone); neurotransmitter markers (low serotonin and noradrenaline); metabolic (low high-density lipoprotein cholesterol) and neurotrophic factors (reduced S100 calcium-binding protein B). Further to this, other reviews have reported on associations between additional biomarkers and treatment 
Table I Overview of recent insights into biomarkers for depression

\begin{tabular}{|c|c|c|c|}
\hline $\begin{array}{l}\text { Biomarker } \\
\text { system }\end{array}$ & Review topic/summary & References & $\begin{array}{l}\text { Evidence } \\
\text { strength* }\end{array}$ \\
\hline \multirow[t]{4}{*}{ Inflammation } & Proinflammatory markers are higher in depression than controls & Haapakoski et $\mathrm{al}^{40}$ & Strong \\
\hline & Inflammation tends to decrease with antidepressant treatment & Hiles et $\mathrm{a}^{50}$ & Medium \\
\hline & Inflammation seems more aberrant in treatment nonresponders & Strawbridge et $\mathrm{al}^{43}$ & Medium \\
\hline & Anti-inflammatory treatments reduce depression severity & Köhler et al ${ }^{178}$ & Strong \\
\hline \multirow[t]{5}{*}{ Neuroendocrine } & HPA axis appears overactive in people with depression & Horowitz and & Strong \\
\hline & & Zunszain 190 & \\
\hline & Atypical depression may show hypocortisolism & Juruena and Cleare ${ }^{191}$ & Medium \\
\hline & High cortisol may predict a poorer response to psychological therapy and & Fischer et al ${ }^{107}$ & Medium \\
\hline & pharmacologic therapy & Anacker et al ${ }^{108}$ & \\
\hline \multirow[t]{3}{*}{ GF } & $\begin{array}{l}\text { Some neurotrophic factors are reduced in depression compared to controls (BDNF, } \\
\text { NGF, GDNF) }\end{array}$ & Molendijk et a $\mathrm{a}^{70}$ & Strong \\
\hline & Some GFs may be overproduced in depression (VEGF, bFGF) & Tseng et $\mathrm{al}^{77}$ & Medium \\
\hline & Neurotrophic factors appear to increase alongside treatment, regardless of response & Castrén and Kojima ${ }^{192}$ & Medium \\
\hline \multirow[t]{2}{*}{ Neurotransmitter } & $\begin{array}{l}\text { There is widespread increased } 5 \text {-HTIA binding in people with depression that can be } \\
\text { influenced by treatment }\end{array}$ & Kaufman et $\mathrm{al}^{96}$ & Strong \\
\hline & $\begin{array}{l}\text { Monoamines interact to influence cognitive function and responses to stress; may provide } \\
\text { mechanisms of TRD }\end{array}$ & Coplan et al ${ }^{99}$ & Medium \\
\hline \multirow[t]{3}{*}{ Metabolic } & Depression is associated with altered metabolic profiles & Pan et al ${ }^{193}$ & Medium \\
\hline & $\begin{array}{l}\text { The promise of metabolic markers for improving depression treatments is limited by the } \\
\text { confounders BMI and severity }\end{array}$ & Carvalho et al ${ }^{194}$ & Medium \\
\hline & Atypical depression linked with greater metabolic abnormalities & Lamers et $\mathrm{al}^{156}$ & Strong \\
\hline
\end{tabular}

Notes: *Strength of evidence coded as follows: weak, medium, strong, very strong, rated as per the extent of inconsistency between findings and indicated promise for the future of this topic. Neuroimaging (eg, Wise et al ${ }^{195}$ ) and genetic markers (Tamatam et al ${ }^{196}$ ) are reviewed extensively elsewhere.

Abbreviations: HPA, hypothalamic-pituitary-adrenal; GF, growth factor; BDNF, brain-derived neurotrophic factor; NGF, nerve growth factor; GDNF, glial cell line-derived neurotrophic factor; VEGF, vascular endothelial growth factor; bFGF, basic fibroblast growth factor; 5-HTIA, serotonin IA receptor; TRD, treatment-resistant depression; BMI, body mass index.

outcomes. ${ }^{19,28-30}$ A brief description of putative markers in each system is outlined in the subsequent sections and in Table 2 .

\section{Inflammatory findings in depression}

Since Smith's seminal paper outlining the macrophage hypothesis, ${ }^{31}$ this established literature has found increased levels of various proinflammatory markers in depressed patients, which have been reviewed widely. ${ }^{32-37}$ Twelve inflammatory proteins have been evaluated in meta-analyses comparing depressed and healthy control populations. ${ }^{38-43}$

IL-6 ( $P<0.001$ in all meta-analyses; 31 studies included $)$ and CRP $(P<0.001 ; 20$ studies $)$ appear frequently and reliably elevated in depression. ${ }^{40}$ Elevated tumor necrosis factor alpha (TNF $\alpha$ ) was identified in early studies $(P<0.001),{ }^{38}$ but substantial heterogeneity rendered this inconclusive when accounting for more recent investigations (31 studies). ${ }^{40}$ IL-1 $\beta$ is even more inconclusively associated with depression, with meta-analyses suggesting higher levels in depression $(P=0.03),{ }^{41}$ high levels only in European studies ${ }^{42}$ or no differences from controls. ${ }^{40}$ Despite this, a recent article suggested particular translational implications for IL- $1 \beta,{ }^{44}$ supported by an extremely significant effect of elevated IL-1 $\beta$ ribonucleic acid predicting a poor response to antidepressants; ${ }^{45}$ other findings above pertain to circulating blood-derived cytokines. The chemokine monocyte chemoattractant protein-1 has shown elevations in depressed participants in one meta-analysis. ${ }^{39}$ Interleukins IL-2, IL-4, IL-8, IL-10 and interferon gamma were not significantly different between depressed patients and controls at a meta-analytic level, but have nonetheless demonstrated potential in terms of altering with treatment: IL-8 has been reported as elevated in those with severe depression prospectively and cross-sectionally, ${ }^{46}$ different patterns of change in IL-10 and interferon gamma during treatment have occurred between early responders versus nonresponders, ${ }^{47}$ while IL-4 and IL-2 have decreased in line with symptom remission. ${ }^{48}$ In meta-analyses, small decreases alongside treatment have been demonstrated for IL-6, IL-1 $\beta$, IL-10 and CRP. ${ }^{43,49,50}$ Additionally, TNF $\alpha$ may only reduce with treatment in responders, and a composite marker index may indicate increased inflammation in patients who subsequently do not respond to treatment. ${ }^{43}$ It is notable, however, that almost all of the research examining inflammatory proteins and treatment response utilize pharmacologic treatment trials. Thus, at least some inflammatory alterations during treatment are likely attributable to antidepressants. The precise inflammatory effects of different antidepressants 
Table 2 Biomarkers with potential translational use for depression

\begin{tabular}{|c|c|c|}
\hline Source/system & Biomarker(s) with potential & References \\
\hline \multirow[t]{7}{*}{ Inflammation } & IL-6, CRP & Haapakoski et al ${ }^{40}$ \\
\hline & TNF $\alpha$ & Strawbridge et al ${ }^{43}$ \\
\hline & IL-I $\beta$ & Farooq et $\mathrm{al}^{44}$ \\
\hline & IL-2, IL-4, IL-I0, IFN $\gamma$ & Dowlati et $\mathrm{al}^{38}$ \\
\hline & IL-8, MCPI & Eyre et $\mathrm{al}^{39}$ \\
\hline & IL-Ia, IFN $\alpha$, IL-5, IL-7, IL-I2, IL-12p70, IL-13, IL-I5, IL-16, IL-17, TNF $\beta$, MCP4, & Novel markers \\
\hline & MipI $\alpha$, MipI $\beta$, SAA, sICAMI, sVCAMI, eotaxin, eotaxin3, TARC, IP-I0, GM-CSF & \\
\hline \multirow[t]{6}{*}{ Growth factors } & BDNF & Molendijk et a $\mathrm{l}^{70}$ \\
\hline & VEGF & Carvalho et $\mathrm{al}^{76}$ \\
\hline & NGF & Chen et $\mathrm{al}^{73}$ \\
\hline & GDNF & Lin and Tseng ${ }^{74}$ \\
\hline & IGF-I & Tu et $\mathrm{a}^{83}$ \\
\hline & bFGF, Tie2, sFltl, PIGF, VEGFC, VEGFD, proBDNF & Novel markers \\
\hline \multirow[t]{3}{*}{ Neurotransmitters } & $5-\mathrm{HT}$ and receptors & Kaufman et al ${ }^{96}$ \\
\hline & NA, DA, glutamate/glutamine, GABA, histamine, MHPG, HVA & Coplan et $\mathrm{a}^{99}$ \\
\hline & & Yoshimura et al ${ }^{197}$ \\
\hline \multirow[t]{3}{*}{ Endocrine } & Cortisol (various measurements) & Fischer et $\mathrm{al}^{107}$ \\
\hline & ACTH, CRH, DHEA, vasopressin & Pierscionek et al ${ }^{198}$ \\
\hline & TSH & Hage and Azar ${ }^{199}$ \\
\hline \multirow[t]{7}{*}{ Metabolic factors } & Leptin & $\mathrm{Lu}^{88}$ \\
\hline & Ghrelin & Wittekind and \\
\hline & & Kluge $^{89}$ \\
\hline & Insulin & Kan et a $\left.\right|^{90}$ \\
\hline & Albumin & Maes et $\mathrm{al}^{93}$ \\
\hline & Glucose & Lustman et al ${ }^{92}$ \\
\hline & Lipids & Liu et $\mathrm{al}^{91}$ \\
\hline Neuroimaging & Structural, for example, gray/white matter volume & Wise et al ${ }^{195}$ \\
\hline markers & Functional, for example, BOLD; PET ligands assessing various sources & Fu et $\mathrm{a}^{29}$ \\
\hline \multirow[t]{4}{*}{ Genetic } & GWAS/polygenic risk & Ripke et al ${ }^{114}$ \\
\hline & Telomere length & Lewis $^{116}$ \\
\hline & Set of candidate gene polymorphisms; epigenetic changes to DNA & Dunn et $\mathrm{a}^{200}$ \\
\hline & (eg, methylation), histone modification; gene expression assessments & \\
\hline
\end{tabular}

Notes: Nonexhaustive collection of biomarkers; many markers across systems can be observed centrally or peripherally, and at multiple levels (eg, proteomic, transcriptomic as well as genetic); see also accelerometer-based measurements, such as of circadian rhythms. ${ }^{201}$

Abbreviations: IL-6, interleukin-6; CRP, C-reactive protein; TNF $\alpha$, tumor necrosis factor alpha; IL-I $\beta$, interleukin-Ibeta; IL-2, interleukin-2; IL-4, interleukin-4; IL-I0, interleukin-I0; IFN $\gamma$, interferon gamma; IL-8, interleukin-8 (CXCL8); MCPI, monocyte chemoattractant protein; IL-I $\alpha$, interleukin-Ialpha; IFN $\alpha$, interferon alpha; IL-5, interleukin-5; IL-7, interleukin-7; IL-12, interleukin-I2; IL-I2p70, interleukin-12p70; IL-I3, interleukin-I3; IL-I5, interleukin-I5; IL-I6, interleukin-I6; IL-I7, interleukin-I7; TNF $\beta$, tumor necrosis factor beta; MCP4, monocyte chemoattractant protein 4; Mipl $\alpha$, macrophage inflammatory protein-Ialpha; MipI $\beta$, macrophage inflammatory protein-I beta; SAA, serum amyloid A; sICAMI, soluble intercellular adhesion molecule I; sVCAMI, soluble vascular cell adhesion molecule I; TARC, thymus and activationregulated chemokine; IP-I0, interferon gamma-induced protein I0; GM-CSF, granulocyte macrophage colony-stimulating factor; BDNF, brain-derived neurotrophic factor; VEGF, vascular endothelial growth factor; NGF, nerve growth factor; GDNF, glial cell line-derived neurotrophic factor; IGF-I, insulin-like growth factor-I; bFGF, basic fibroblast growth factor; Tie2, tyrosine-kinase2; sFlt-I, soluble fms-like tyrosine kinase-I; PIGF, placental growth factor; 5-HT, 5-hydroxytryptamine; NA, noradrenaline; DA, dopamine; GABA, gamma-aminobutyric acid; MHPG, 3-methoxy-4-hydroxyphenylglycol; HVA, homovanillic acid; ACTH, adrenocorticotropin hormone; CRH, corticotrophin-releasing hormone; DHEA, dehydroepiandrosterone; TSH, thyroid-stimulating hormone; BOLD, blood oxygen level dependent; PET, positron emission tomography; GWAS, genome-wide association study.

have not yet been established, but evidence using CRP levels suggests individuals respond differently to specific treatments based on baseline inflammation: Harley et $\mathrm{al}^{51}$ reported elevated pretreatment CRP predicting a poor response to psychological therapy (cognitive-behavioral or interpersonal psychotherapy), but a good response to nortriptyline or fluoxetine; Uher et $\mathrm{al}^{52}$ replicated this finding for nortriptyline and identified the opposite effect for escitalopram. In contrast, Chang et $\mathrm{al}^{53}$ found higher CRP in early responders to fluoxetine or venlafaxine than nonresponders. Furthermore, patients with TRD and high CRP have responded better to the TNF $\alpha$ antagonist infliximab than those with levels in the normal range. ${ }^{54}$

Together, the evidence suggests that even when controlling for factors such as body mass index (BMI) and age, inflammatory responses appear aberrant in approximately one-third of patients with depression. ${ }^{55,56}$ The inflammatory system, however, is extremely complex, and there are numerous biomarkers representing different aspects of this system. Recently, additional novel cytokines and chemokines have yielded evidence of abnormalities in depression. These include: macrophage inhibitory protein 1a, IL-1a, IL-7, 
IL-12p70, IL-13, IL-15, eotaxin, granulocyte macrophage colony-stimulating factor, ${ }^{57} \mathrm{IL}-5,{ }^{58} \mathrm{IL}-16,{ }^{59} \mathrm{IL}-17,{ }^{60}$ monocyte chemoattractant protein- $4,{ }^{61}$ thymus and activationregulated chemokine, ${ }^{62}$ eotaxin-3, TNFb, ${ }^{63}$ interferon gamma-induced protein $10,{ }^{64}$ serum amyloid A, ${ }^{65}$ soluble intracellular adhesion molecule ${ }^{66}$ and soluble vascular cell adhesion molecule $1 .^{67}$

\section{Growth factor findings in depression}

In light of the potential importance of non-neurotrophic growth factors (such as those relating to angiogenesis), we refer to neurogenic biomarkers under the broader definition of growth factors.

Brain-derived neurotrophic factor (BDNF) is the most frequently studied of these. Multiple meta-analyses demonstrate attenuations of the BDNF protein in serum, which appear to increase alongside antidepressant treatment. ${ }^{68-71}$ The most recent of these analyses suggests that these BDNF aberrations are more pronounced in the most severely depressed patients, but that antidepressants appear to increase the levels of this protein even in the absence of clinical remission. ${ }^{70}$ proBDNF has been less widely studied than the mature form of BDNF, but the two appear to differ functionally (in terms of their effects on tyrosine receptor kinase B receptors) and recent evidence suggests that while mature BDNF may be reduced in depression, proBDNF may be overproduced. ${ }^{72}$ Nerve growth factor assessed peripherally has also been reported as lower in depression than in controls in a meta-analysis, but may not be altered by antidepressant treatment despite being most attenuated in patients with more severe depression. ${ }^{73}$ Similar findings have been reported in a meta-analysis for glial cell line-derived neurotrophic factor. ${ }^{74}$

Vascular endothelial growth factor (VEGF) has a role in promoting angiogenesis and neurogenesis along with other members of the VEGF family (eg, VEGF-C, VEGF-D) and has promise for depression. ${ }^{75}$ Despite inconsistent evidence, two meta-analyses have recently indicated elevations of VEGF in blood of depressed patients compared to controls (across 16 studies; $P<0.001$ ). ${ }^{76,77}$ However, low VEGF has been identified in $\mathrm{TRD}^{78}$ and higher levels have predicted nonresponse to antidepressant treatment. ${ }^{79}$ It is not understood why the levels of VEGF protein would be elevated, but it may partly be attributable to proinflammatory activity and/or increases in blood-brain barrier permeability in depressed states that causes reduced expression in cerebrospinal fluid. ${ }^{80}$ The relationship between VEGF and treatment response is unclear; a recent study found no relationship between either serum VEGF or BDNF with response or depression severity, despite decreases alongside antidepressant treatment. ${ }^{81}$ Insulin-like growth factor-1 is an additional factor with neurogenic functions that may be increased in depression, reflecting an imbalance in neurotrophic processes. ${ }^{82,83}$ Basic fibroblast growth factor (or FGF-2) is a member of the fibroblast growth factor family and appears higher in depressed than control groups. ${ }^{84}$ However, reports are not consistent; one found that this protein was lower in MDD than healthy controls, but reduced further alongside antidepressant treatment. ${ }^{85}$

Further growth factors that have not been sufficiently explored in depression include tyrosine kinase 2 and soluble fms-like tyrosine kinase-1 (also termed sVEGFR-1) which act in synergy with VEGF, and tyrosine kinase receptors (that bind BDNF) may be attenuated in depression. ${ }^{86}$ Placental growth factor is also part of the VEGF family, but has not been studied in systematically depressed samples to our knowledge.

\section{Metabolic biomarker findings in depression}

The main biomarkers associated with metabolic illness include leptin, adiponectin, ghrelin, triglycerides, highdensity lipoprotein (HDL), glucose, insulin and albumin. ${ }^{87}$ The associations between many of these and depression have been reviewed: leptin ${ }^{88}$ and ghrelin ${ }^{89}$ appear lower in depression than controls in the periphery and may increase alongside antidepressant treatment or remission. Insulin resistance may be increased in depression, albeit by small amounts. ${ }^{90}$ Lipid profiles, including HDL-cholesterol, appear altered in many patients with depression, including those without comorbid physical illness, though this relationship is complex and requires further elucidation. ${ }^{91}$ Additionally, hyperglycemia ${ }^{92}$ and hypoalbuminemia ${ }^{93}$ in depression have been reported in reviews.

Investigations of overall metabolic states are becoming more frequent using metabolomics panels of small molecules with the hope of finding a robust biochemical signature for psychiatric disorders. In a recent study using artificial intelligence modeling, a set of metabolites illustrating increased glucose-lipid signaling was highly predictive of an MDD diagnosis, ${ }^{94}$ supportive of previous studies. ${ }^{95}$

\section{Neurotransmitter findings in depression}

While the attention paid to monoamines in depression has yielded relatively successful treatments, no robust neurotransmitter markers have been identified to optimize treatment based on the selectivity of monoamine targets of antidepressants. Recent work points toward the serotonin 
(5-hydroxytryptamine) 1A receptor as potentially important for both diagnosis and prognosis of depression, pending new genetic and imaging techniques. ${ }^{96}$ There are new potential treatments targeting 5-hydroxytryptamine; for example, using a slow-release administration of 5-hydroxytryptophan. ${ }^{97}$ Increased transmission of dopamine interacts with other neurotransmitters to improve cognitive outcomes such as decision making and motivation. ${ }^{98}$ Similarly, the neurotransmitters glutamate, noradrenaline, histamine and serotonin may interact and activate as part of a depression-related stress response; this might decrease 5-hydroxytryptamine production through "flooding". A recent review sets out this theory and suggests that in TRD, this could be reversed (and 5-HT restored) through multimodal treatment targeting multiple neurotransmitters. ${ }^{99}$ Interestingly, increases in serotonin do not always occur conjunctively with therapeutic antidepressant benefits. ${ }^{100}$ Despite this, neurotransmitter metabolites such as 3-methoxy-4-hydroxyphenylglycol, of noradrenaline, or homovanillic acid, of dopamine, have often been found to increase alongside reduction in depression with antidepressant treatment ${ }^{101,102}$ or that low levels of these metabolites predict a better response to SSRI treatment. ${ }^{102,103}$

\section{Neuroendocrine findings in depression}

Cortisol is the most common HPA axis biomarker to have been studied in depression. Numerous reviews have focused on the various assessments of HPA activity; overall, these suggest that depression is associated with hypercortisolemia and that the cortisol awakening response is often attenuated. ${ }^{104,105}$ This is supported by a recent review of chronic cortisol levels measured in hair, supporting the hypothesis of cortisol hyperactivity in depression but hypoactivity in other illnesses such as panic disorder. ${ }^{106}$ Furthermore, particularly, elevated cortisol levels may predict a poorer response to psychological ${ }^{107}$ and antidepressant ${ }^{108}$ treatment. Historically, the most promising neuroendocrine marker of prospective treatment response has been the dexamethasone suppression test, where cortisol nonsuppression following dexamethasone administration is associated with a lower likelihood of subsequent remission. However, this phenomenon has not been considered sufficiently robust for clinical application. Related markers corticotrophin-releasing hormone and adrenocorticotropin hormone as well as vasopressin are inconsistently found to be overproduced in depression and dehydroepiandrosterone is found to be attenuated; the ratio of cortisol to dehydroepiandrosterone may be elevated as a relatively stable marker in TRD, persisting after remission. ${ }^{109}$ Neuroendocrine hormone dysfunctions have long been associated with depression, and hypothyroidism may also play a causal role in depressed mood. ${ }^{110}$ Furthermore, thyroid responses can normalize with successful treatment for depression. ${ }^{111}$

Within the above, it is important also to consider signaling pathways across systems, such as glycogen synthase kinase-3, mitogen-activated protein kinase and cyclic adenosine $3^{\prime}, 5^{\prime}$-monophosphate, involved in synaptic plasticity ${ }^{112}$ and modified by antidepressants. ${ }^{113}$ Further potential biomarker candidates that span biologic systems particularly are measured using neuroimaging or genetics. In response to the lack of robust and meaningful genomic differences between depressed and nondepressed populations, ${ }^{114}$ novel genetic approaches such as polygenic scores ${ }^{115}$ or telomere length ${ }^{116,117}$ could prove more useful. Additional biomarkers gaining popularity are examining circadian cycles or chronobiologic biomarkers utilizing different sources. Actigraphy can provide an objective assessment of sleep and wake activity and rest through an accelerometer, and actigraphic devices can increasingly measure additional factors such as light exposure. This may be more useful for detection than commonly used subjective reports of patients and could provide novel predictors of treatment response. ${ }^{118}$ The question of which biomarkers are the most promising for translational use is a challenging one, which is expanded upon below.

\section{Current challenges}

For each of these five neurobiological systems reviewed, the evidence follows a similar narrative: there are many biomarkers that exist that are associated in some respects with depression. These markers are frequently interrelated in a complex, difficult-to-model fashion. The evidence is inconsistent, and it is likely that some are epiphenomena of other factors and some are important in only a subset of patients. Biomarkers are likely to be useful through a variety of routes (eg, those that predict subsequent response to treatment, those indicating specific treatments as more likely to be effective or those that alter with interventions regardless of clinical improvements). Novel methods are required to maximize consistency and clinical applicability of biologic assessments in psychiatric populations.

\section{Biomarker variability}

Variation of biomarkers over time and across situations pertains more to some types (eg, proteomics) than others (genomics). Standardized norms for many do not exist or have not been widely accepted. Indeed, the influence of environmental factors on markers frequently depends on genetic composition and other physiologic differences between 
people that cannot all be accounted for. This makes the assessment of biomarker activity, and identifying biologic abnormalities, difficult to interpret. Due to the number of potential biomarkers, many have not been measured widely or in a complete panel alongside other relevant markers.

Many factors have been reported to alter the protein levels across biologic systems in patients with affective disorders. Along with research-related factors such as duration and conditions of storage (which may cause degradation of some compounds), these include time of day measured, ethnicity, exercise, ${ }^{119}$ diet (eg, microbiome activity, especially provided that most blood biomarker studies do not require a fasting sample), ${ }^{120}$ smoking and substance use, ${ }^{121}$ as well as health factors (such as comorbid inflammatory, cardiovascular or other physical illnesses). For example, although heightened inflammation is observed in depressed but otherwise healthy individuals compared to nondepressed groups, depressed individuals who also have a comorbid immune-related condition frequently have even higher levels of cytokines than either those without depression or illness. ${ }^{122}$ Some prominent factors with probable involvement in the relationship between biomarkers, depression and treatment response are outlined below.

\section{Stress}

Both endocrine and immune responses have well-known roles in responding to stress (physiologic or psychological), and transient stress at the time of biologic specimen collection is rarely measured in research studies despite the variability of this factor between individuals that may be accentuated by current depressive symptoms. Both acute and chronic psychological stressors act as an immune challenge, accentuating inflammatory responses in the short and longer term. ${ }^{123,124}$ This finding extends to the experience of early-life stress, which has been associated with adult inflammatory elevations that are independent of stress experienced as an adult. ${ }^{125,126}$ During childhood traumatic experience, heightened inflammation has also been reported only in those children who were currently depressed. ${ }^{127}$ Conversely, people with depression and a history of childhood trauma may have blunted cortisol responses to stress, compared to those with depression and no early-life trauma. ${ }^{128}$ Stress-induced HPA axis alterations appear interrelated with cognitive function, ${ }^{129}$ as well as depression subtype or variation in HPA-related genes. ${ }^{130}$ Stress also has shortand long-term impairing effects on neurogenesis ${ }^{131}$ and other neural mechanisms. ${ }^{132}$ It is unclear precisely how childhood trauma affects biologic markers in depressed adults, but it is possible that early-life stress predisposes some individuals to enduring stress reactions in adulthood that are amplified psychologically and/or biologically.

\section{Cognitive functioning}

Neurocognitive dysfunctions occur frequently in people with affective disorders, even in unmedicated MDD. ${ }^{133}$ Cognitive deficits appear cumulative alongside treatment resistance. ${ }^{134}$ Neurobiologically, the HPA axis ${ }^{129}$ and neurotrophic systems ${ }^{135}$ are likely to play a key role in this relationship. Neurotransmitters noradrenaline and dopamine are likely important for cognitive processes such as learning and memory. ${ }^{136}$ Elevated inflammatory responses have been linked with cognitive decline, and likely affect cognitive functioning in depressive episodes, ${ }^{137}$ and in remission, through a variety of mechanisms. ${ }^{138}$ Indeed, Krogh et al ${ }^{139}$ proposed that CRP is more closely related to cognitive performance than to the core symptoms of depression.

\section{Age, gender and BMI}

The absence or presence, and direction of biologic differences between men and women has been particularly variable in the evidence to date. Neuroendocrine hormone variation between men and women interacts with depression susceptibility. ${ }^{140}$ A review of inflammation studies reported that controlling for age and gender did not affect patient-control differences in inflammatory cytokines (although the association between IL-6 and depression reduced as age increased, which is consistent with theories that inflammation generally heightens with age). ${ }^{41,141}$ VEGF differences between patients and controls are larger in studies assessing younger samples, while gender, BMI and clinical factors did not affect these comparisons at a meta-analytic level. ${ }^{77}$ However, the lack of adjustment for BMI in previous examinations of inflammation and depression appears to confound highly significant differences reported between these groups. ${ }^{41}$ Enlarged adipose tissue has been definitively demonstrated to stimulate cytokine production as well as being closely linked to metabolic markers. ${ }^{142}$ Because psychotropic medications may be associated with weight gain and a higher BMI, and these have been associated with treatment resistance in depression, this is an important area to examine.

\section{Medication}

Many biomarker studies in depression (both cross-sectional and longitudinal) have collected baseline specimens in unmedicated participants to reduce heterogeneity. However, many of these assessments are taken after a wash-out period from medication, which leaves the potentially significant 
confounding factor of residual changes in physiology, exacerbated by the extensive range of treatments available that may have had differing effects on inflammation. Some studies have excluded psychotropic, but not other medication use: in particular, the oral contraceptive pill is frequently permitted in research participants and not controlled for in analyses, which has recently been indicated to increase hormone and cytokine levels. ${ }^{143,144}$ Several studies indicate that antidepressant medications have effects on the inflammatory response, ${ }^{34,43,49,145-147}$ HPA-axis, ${ }^{108}$ neurotransmitter, ${ }^{148}$ and neurotrophic ${ }^{149}$ activity. However, the numerous potential treatments for depression have distinct and complex pharmacologic properties, suggesting there may be discrete biologic effects of different treatment options, supported by current data. It has been theorized that in addition to monoamine effects, specific serotonin-targeting medications (ie, SSRIs) are likely to target $\mathrm{Th} 2$ shifts in inflammation, and noradrenergic antidepressants (eg, SNRIs) effect a Th1 shift. ${ }^{150}$ It is not yet possible to determine the effects of individual or combination medications on biomarkers. These are likely mediated by other factors including the length of treatment (few trials assess long-term medication use), sample heterogeneity and not stratifying participants by response to treatment.

\section{Heterogeneity Methodological}

As alluded to above, differences (between and within studies) in terms of which treatments (and combinations) the participants are taking and have taken previously are bound to introduce heterogeneity into research findings, particularly in biomarker research. In addition to this, many other design and sample characteristics vary across studies, thus augmenting the difficulty with interpreting and attributing findings. These include biomarker measurement parameters (eg, assay kits) and methods of collecting, storing, processing and analyzing markers in depression. Hiles et $\mathrm{al}^{141}$ examined some sources of inconsistency in the literature on inflammation and found that accuracy of depression diagnosis, BMI and comorbid illnesses were most important to account for in assessing peripheral inflammation between depressed and nondepressed groups.

\section{Clinical}

The extensive heterogeneity of depressed populations is well documented $^{151}$ and is a critical contributor to contrasting findings within the research literature. It is probable that even within diagnoses, abnormal biologic profiles are confined to subsets of individuals that may not be stable over time.
Cohesive subgroups of people suffering with depression may be identifiable through a combination of psychological and biologic factors. Below, we outline the potential for exploring subgroups in meeting the challenges that biomarker variability and heterogeneity pose.

\section{Subtypes within depression}

Thus far, no homogenous subgroups within depression episodes or disorders have been reliably able to distinguish between patients based on symptom presentations or treatment responsiveness. ${ }^{152}$ The existence of a subgroup in whom biologic aberrations are more pronounced would help to explain the heterogeneity between previous studies and could catalyze the path toward stratified treatment. Kunugi et al ${ }^{153}$ have proposed a set of four potential subtypes based on the role of different neurobiological systems displaying clinically relevant subtypes in depression: those with hypercortisolism presenting with melancholic depression, or hypocortisolism reflecting an atypical subtype, a dopamine-related subset of patients who may present prominently with anhedonia (and could respond well to, eg, aripiprazole) and an inflammatory subtype characterized by elevated inflammation. Many articles focusing on inflammation have specified the case for the existence of an "inflammatory subtype" within depression. ${ }^{55,56,154,155}$ Clinical correlates of elevated inflammation are as yet undetermined and few direct attempts have been made to discover which participants may comprise this cohort. It has been proposed that people with atypical depression could have higher levels of inflammation than the melancholic subtype, ${ }^{156}$ which is perhaps not in line with findings regarding the HPA axis in melancholic and atypical subtypes of depression. TRD ${ }^{37}$ or depression with prominent somatic symptoms ${ }^{157}$ has also been posited as a potential inflammatory subtype, but neurovegetative (sleep, appetite, libido loss), mood (including low mood, suicidality and irritability) and cognitive symptoms (including affective bias and guilt) $)^{158}$ all appear related to biologic profiles. Further potential candidates for an inflammatory subtype involve the experience of sickness behavior-like symptoms ${ }^{159,160}$ or a metabolic syndrome. ${ }^{158}$

The propensity toward (hypo) mania may distinguish biologically between patients suffering from depression. Evidence now suggests that bipolar illnesses are a multifaceted group of mood disorders, with bipolar subsyndromal disorder found more prevalently than was previously recognized. ${ }^{161}$ Inaccurate and/or delayed detection of bipolar disorder has recently been highlighted as a major problem in clinical psychiatry, with the average time to correct diagnosis frequently 
exceeding a decade ${ }^{162}$ and this delay causing greater severity and cost of overall illness. ${ }^{163}$ With the majority of patients with bipolar disorder presenting initially with one or more depressive episodes and unipolar depression being the most frequent misdiagnosis, the identification of factors that might differentiate between unipolar and bipolar depression has substantial implications. ${ }^{164}$ Bipolar spectrum disorders likely have been undetected in some previous MDD biomarker investigations, and smatterings of evidence have indicated differentiation of HPA axis activity ${ }^{109}$ or inflammation ${ }^{165,166}$ between bipolar and unipolar depression. However, these comparisons are scarce, possess small sample sizes, identified nonsignificant trend effects or recruited populations that were not well characterized by diagnosis. These investigations also do not examine the role of treatment responsiveness in these relationships.

Both bipolar disorders ${ }^{167}$ and treatment resistance ${ }^{168}$ are not dichotomous constructs and lie on continua, which increases the challenge of subtype identification. Apart from subtyping, it is worth noting that many biologic abnormalities observed in depression are similarly found in patients with other diagnoses. Thus, transdiagnostic examinations are also potentially important.

\section{Biomarker measurement challenges}

\section{Biomarker selection}

The large number of potentially useful biomarkers presents a challenge for psychobiology in determining which markers are implicated in which way and for whom. To increase the challenge, relatively few of these biomarkers have been subject to sufficient investigation in depression, and for most, their precise roles in healthy and clinical populations are not well understood. Despite this, a number of attempts have been made to propose promising biomarker panels. In addition to Brand et al's 16 sets of markers with strong potential, ${ }^{27}$ Lopresti et al outline an additional extensive set of oxidative stress markers with potential for improving treatment response. ${ }^{28}$ Papakostas et al defined a priori a set of nine serum markers spanning biologic systems (BDNF, cortisol, soluble TNF $\alpha$ receptor type II, alphal antitrypsin, apolipoprotein CIII, epidermal growth factor, myeloperoxidase, prolactin and resistin) in validation and replication samples with MDD. Once combined, a composite measure of these levels was able to distinguish between MDD and control groups with $80 \%-90 \%$ accuracy. ${ }^{169}$ We propose that even these do not cover all potential candidates in this field; see Table 2 for a nonexhaustive delineation of biomarkers with potential for depression, containing both those with an evidence base and promising novel markers.

\section{Technology}

Due to technologic advances, it is now possible (indeed, convenient) to measure a large array of biomarkers simultaneously at a lower cost and with higher sensitivity than has been the case previously. At present, this capability to measure numerous compounds is ahead of our ability to effectively analyze and interpret the data, ${ }^{170}$ something that will continue with the rise in biomarker arrays and new markers such as with metabolomics. This is largely due to a lack of understanding about the precise roles of and the interrelationships between markers, and an insufficient grasp of how related markers associate across different biologic levels (eg, genetic, transcription, protein) within and between individuals. Big data using new analytical approaches and standards will assist with addressing this, and new methodologies are being proposed; one example is the development of a statistical approach grounded in flux-based analysis to discover new potential metabolic markers based on their reactions between networks and integrate gene expression with metabolite data. ${ }^{171}$ Machine learning techniques are already being applied and will assist with models using biomarker data to predict treatment outcomes in studies with big data. ${ }^{172}$

\section{Aggregating biomarkers}

Examining an array of biomarkers simultaneously is an alternative to inspecting isolated markers that could provide a more accurate viewpoint into the complex web of biologic systems or networks. ${ }^{26}$ Also, to assist with disentangling contrasting evidence in this literature to date (particularly, where biomarker networks and interactions are well understood), biomarker data can then be aggregated or indexed. One challenge is in identifying the optimum method of conducting this, and it may require enhancements in technology and/or novel analytical techniques (see the "Big data" section). Historically, ratios between two distinct biomarkers have yielded interesting findings. ${ }^{109,173}$ Few attempts have been made to aggregate biomarker data on a larger scale, such as those using principal component analysis of proinflammatory cytokine networks. ${ }^{174}$ In a meta-analysis, proinflammatory cytokines have been converted into a single-effect size score for each study, and overall showed significantly higher inflammation before antidepressant treatment, predicting subsequent nonresponse in outpatient studies. Composite biomarker panels are both a challenge and opportunity for future research to identify meaningful and reliable findings that can be applied to improve treatment outcomes. ${ }^{43}$ A study by Papakostas et al took an alternative approach, selecting a panel of heterogeneous serum biomarkers (of inflammatory, 
HPA axis and metabolic systems) that had been indicated to differ between depressed and control individuals in a previous study and composited these into a risk score which differed in two independent samples and a control group with $>80 \%$ sensitivity and specificity. ${ }^{169}$

\section{Big data}

The use of big data is probably necessary for addressing the current challenges outlined surrounding heterogeneity, biomarker variability, identifying the optimal markers and bringing the field toward translational, applied research in depression. However, as outlined above, this brings technological and scientific challenges. ${ }^{175}$ The health sciences have only recently begun using big data analytics, a decade or so later than in the business sector. However, studies such as iSPOT-D ${ }^{152}$ and consortia such as the Psychiatric Genetics Consortium ${ }^{176}$ are progressing with our understanding of biologic mechanisms in psychiatry. Machine-learning algorithms have, in very few studies, started to be applied to biomarkers for depression: a recent investigation pooled data from $>5,000$ participants of 250 biomarkers; after multiple imputation of data, a machine-learning boosted regression was conducted, indicating 21 potential biomarkers. Following further regression analyses, three biomarkers were selected as associating most strongly with depressive symptoms (highly variable red blood cell size, serum glucose and bilirubin levels). The authors conclude that big data can be used effectively for generating hypotheses. ${ }^{177}$ Larger biomarker phenotyping projects are now underway and will help to advance our journey into the future of the neurobiology of depression.

\section{Future prospects}

\section{Biomarker panel identification}

The findings in the literature to date require replication in large-scale studies. This is particularly true for novel biomarkers, such as the chemokine thymus and activationregulated chemokine and the growth factor tyrosine kinase 2 which, to our knowledge, have not been investigated in clinically depressed and healthy control samples. Big data studies must assay comprehensive biomarker panels and use sophisticated analysis techniques to fully ascertain the relationships between markers and those factors which modify them in clinical and nonclinical populations. Additionally, large-scale replications of principal component analysis might establish highly correlated groups of biomarkers and could also inform the use of "composites" in biologic psychiatry, which may enhance the homogeneity of future findings.

\section{Discovery of homogenous subtypes}

Regarding biomarker selection, multiple panels may be required for different potential pathways that research could implicate. Taken together, the current evidence indicates that biomarker profiles are assuredly, but abstrusely altered in a subpopulation of individuals currently suffering from depression. This may be established within or across diagnostic categories, which would account for some inconsistency of findings that can be observed in this literature. Quantifying a biologic subgroup (or subgroups) may most effectively be facilitated by a large cluster analysis of biomarker network panels in depression. This would illustrate within-population variability; latent class analyses could exhibit distinct clinical characteristics based on, for example, inflammation.

\section{Specific treatment effects on inflammation and response}

All commonly prescribed treatments for depression should be comprehensively assessed for their specific biologic effects, also accounting for the effectiveness of treatment trials. This may enable constructs relating to biomarkers and symptom presentations to predict outcomes to a variety of antidepressant treatments in a more personalized fashion, and may be possible in the context of both unipolar and bipolar depression. This is likely to be useful for new potential treatments as well as currently indicated treatments.

\section{Prospective determination of treatment response}

Use of the above techniques is likely to result in an improved ability to forecast treatment resistance prospectively. More authentic and persistent (eg, long-term) measures of treatment response may contribute to this. Assessment of other valid measures of patient well-being (such as quality of life and everyday functioning) could provide a more holistic assessment of treatment outcome that may associate more closely with biomarkers. While biologic activity alone might not be able to distinguish treatment responders from nonresponders, concurrent measurement of biomarkers with psychosocial or demographic variables could be integrated with biomarker information in developing a predictive model of insufficient treatment response. If a reliable model is developed to predict response (either for the depressed population or a subpopulation) and is validated retrospectively, a translational design can establish its applicability in a large controlled trial.

\section{Toward stratified treatments}

At present, patients with depression are not systematically directed to receive an optimized intervention program. 
If validated, a stratified trial design could be employed to test a model to predict nonresponse and/or to determine where a patient needs to be triaged in a stepped care model. This could be useful in both standardized and naturalistic treatment settings, across different types of intervention. Ultimately, a clinically viable model could be developed to provide individuals with the most appropriate treatment, to recognize those who are likely to develop refractory depression and supply enhanced care and monitoring to these patients. Patients identified as being at risk for treatment resistance may be prescribed a concomitant psychological and pharmacologic therapy or combination pharmacotherapy. As a speculative example, participants with no proinflammatory cytokine elevations might be indicated to receive psychological rather than pharmacologic therapy, while a subset of patients with particularly high inflammation could receive an antiinflammatory agent in augmentation to standard treatment. Similar to stratification, personalized treatment-selection strategies may be possible in the future. For example, a particular depressed individual might have markedly high TNF $\alpha$ levels, but no other biologic abnormalities, and could benefit from short-term treatment with a TNF $\alpha$ antagonist. ${ }^{54}$ Personalized treatment may also entail monitoring biomarker expression during treatment to inform possible intervention changes, the length of continuation therapy required or to detect early markers of relapse.

\section{Novel treatment targets}

There are a huge number of potential treatments that could be effective for depression, which have not been adequately examined, including novel or repurposed interventions from other medical disciplines. Some of the most popular targets have been in anti-inflammatory medications such as celecoxib (and other cyclooxygenase-2 inhibitors), TNF $\alpha$ antagonists etanercept and infliximab, minocycline or aspirin. These appear promising. ${ }^{178}$ Antiglucocorticoid compounds, including ketoconazole ${ }^{179}$ and metyrapone, ${ }^{180}$ have been investigated for depression, but both have drawbacks with their side effect profile and the clinical potential of metyrapone is uncertain. Mifepristone ${ }^{181}$ and the corticosteroids fludrocortisone and spironolactone, ${ }^{182}$ and dexamethasone and hydrocortisone ${ }^{183}$ may also be effective in treating depression in the short term. Targeting glutamate $N$-methyl-D-aspartate receptor antagonists, including ketamine, might represent efficacious treatments in depression. ${ }^{184}$ Omega-3 polyunsaturated fatty acids influence inflammatory and metabolic activity and appear to demonstrate some effectiveness for depression. ${ }^{185}$ It is possible that statins may have antidepressant effects ${ }^{186}$ through relevant neurobiological pathways. ${ }^{187}$
In this way, the biochemical effects of antidepressants (see the "Medication" section) have been utilized for clinical benefits in other disciplines: particularly gastroenterological, neurologic and nonspecific symptom illnesses. ${ }^{188}$ Antiinflammatory effects of antidepressants may represent part of the mechanism for these benefits. Lithium has also been suggested to reduce inflammation, critically through glycogen synthase kinase-3 pathways. ${ }^{189}$ A focus on these effects could prove informative for a depression biomarker signature and, in turn, biomarkers could represent surrogate markers for novel drug development.

\section{Conclusion}

The literature indicates that approximately two-thirds of patients with depression do not achieve remission to an initial treatment and that the likelihood of nonresponse increases with the number of treatments trialed. Providing ineffective therapies has substantial consequences for individual and societal cost, including persistent distress and poor wellbeing, risk of suicide, loss of productivity and wasted health care resources. The vast literature in depression indicates a huge number of biomarkers with the potential to improve treatment for people with depression. In addition to neurotransmitter and neuroendocrine markers which have been subject to widespread study for many decades, recent insights highlight the inflammatory response (and the immune system more generally), metabolic and growth factors as importantly involved in depression. However, excessive contrasting evidence illustrates that there are a number of challenges needing to be tackled before biomarker research can be applied in order to improve the management and care of people with depression. Due to the sheer complexity of biologic systems, simultaneous examinations of a comprehensive range of markers in large samples are of considerable benefit in discovering interactions between biologic and psychological states across individuals. Optimizing the measurement of both neurobiological parameters and clinical measures of depression is likely to facilitate greater understanding. This review also highlights the importance of examining potentially modifying factors (such as illness, age, cognition and medication) in gleaning a coherent understanding of the biology of depression and mechanisms of treatment resistance. It is likely that some markers will show most promise for predicting treatment response or resistance to specific treatments in a subgroup of patients, and the concurrent measurement of biologic and psychological data may enhance the ability to prospectively identify those at risk for poor treatment outcomes. Establishing a biomarker panel has implications for boosting diagnostic accuracy and prognosis, 
as well as for individualizing treatments at the earliest practicable stage of depressive illness and developing effective novel treatment targets. These implications may be confined to subgroups of depressed patients. The pathways toward these possibilities complement recent research strategies to link clinical syndromes more closely to underlying neurobiological substrates. ${ }^{6}$ Apart from reducing heterogeneity, this may facilitate a shift toward parity of esteem between physical and mental health. It is clear that although much work is needed, establishment of the relationship between relevant biomarkers and depressive disorders has substantial implications for reducing the burden of depression at an individual and societal level.

\section{Acknowledgments}

This report represents independent research funded by the National Institute for Health Research (NIHR) Biomedical Research Centre at South London and Maudsley NHS Foundation Trust and King's College London. The views expressed are those of the authors and not necessarily those of the NHS, the NIHR or the Department of Health.

\section{Disclosure}

AHY has in the last 3 years received honoraria for speaking from Astra Zeneca (AZ), Lundbeck, Eli Lilly, Sunovion; honoraria for consulting from Allergan, Livanova and Lundbeck, Sunovion, Janssen; and research grant support from Janssen and UK funding agencies (NIHR, MRC, Wellcome Trust). AJC has in the last 3 years received honoraria for speaking from Astra Zeneca (AZ), honoraria for consulting from Allergan, Livanova and Lundbeck, and research grant support from Lundbeck and UK funding agencies (NIHR, MRC, Wellcome Trust).

The authors report no other conflicts of interest in this work.

\section{References}

1. Prince M, Patel V, Saxena S, et al. No health without mental health. Lancet. 2007;370(9590):859-877.

2. Kingdon D, Wykes T. Increased funding needed for mental health research. BMJ. 2013;346:f402.

3. Vivekanantham S, Strawbridge R, Rampuri R, Ragunathan T, Young AH. Parity of publication for psychiatry. Br J Psychiatry. 2016;209(3): 257-261.

4. Fava M. Diagnosis and definition of treatment-resistant depression. Biol Psychiatry. 2003;53(8):649-659.

5. Insel T, Cuthbert B, Garvey M, et al. Research domain criteria (RDoC): toward a new classification framework for research on mental disorders. Am J Psychiatry. 2010;167(7):748-751.

6. Kapur S, Phillips AG, Insel TR. Why has it taken so long for biological psychiatry to develop clinical tests and what to do about it. Mol Psychiatry. 2012;17(12):1174-1179.
7. Gaynes BN, Warden D, Trivedi MH, Wisniewski SR, Fava M, Rush JA What did STAR*D teach us? Results from a large-scale, practical, clinical trial for patients with depression. Psychiatr Serv. 2009;60(11): 1439-1445.

8. Fekadu A, Rane LJ, Wooderson SC, Markopoulou K, Poon L, Cleare AJ. Prediction of longer-term outcome of treatment-resistant depression in tertiary care. Br J Psychiatry. 2012;201(5):369-375.

9. Fekadu A, Wooderson SC, Markopoulo K, Donaldson C, Papadopoulos A, Cleare AJ. What happens to patients with treatment-resistant depression? A systematic review of medium to long term outcome studies. $J$ Affect Disord. 2009;116(1-2):4-11.

10. Trivedi M. Treatment strategies to improve and sustain remission in major depressive disorder. Dialogues Clin Neurosci. 2008;10(4):377.

11. Fekadu A, Wooderson SC, Markopoulou K, Cleare AJ. The Maudsley Staging Method for treatment-resistant depression: prediction of longerterm outcome and persistence of symptoms. J Clin Psychiatry. 2009; 70(7):952-957.

12. Bennabi D, Aouizerate B, El-Hage W, et al. Risk factors for treatment resistance in unipolar depression: a systematic review. J Affect Disord. 2015;171:137-141.

13. Serretti A, Olgiati P, Liebman MN, et al. Clinical prediction of antidepressant response in mood disorders: linear multivariate vs. neural network models. Psychiatry Res. 2007;152(2-3):223-231.

14. Driessen E, Hollon SD. Cognitive behavioral therapy for mood disorders: efficacy, moderators and mediators. Psychiatr Clin North Am. 2010; 33(3):537-555.

15. Cleare A, Pariante C, Young A, et al; Members of the Consensus Meeting. Evidence-based guidelines for treating depressive disorders with antidepressants: a revision of the 2008 british association for Psychopharmacology guidelines. J Psychopharmacol. 2015;29(5):459-525.

16. Tunnard C, Rane LJ, Wooderson SC, et al. The impact of childhood adversity on suicidality and clinical course in treatment-resistant depression. J Affect Disord. 2014;152-154:122-130.

17. Nemeroff CB, Heim CM, Thase ME, et al. Differential responses to psychotherapy versus pharmacotherapy in patients with chronic forms of major depression and childhood trauma. Proc Natl Acad Sci US A. 2003; 100(24):14293-14296.

18. Nierenberg AA. Predictors of response to antidepressants general principles and clinical implications. Psychiatr Clin North Am. 2003; 26(2):345-352.

19. Thase ME. Using biomarkers to predict treatment response in major depressive disorder: evidence from past and present studies. Dialogues Clin Neurosci. 2014;16(4):539-544.

20. Jani BD, McLean G, Nicholl BI, et al. Risk assessment and predicting outcomes in patients with depressive symptoms: a review of potential role of peripheral blood based biomarkers. Front Hum Neurosci. 2015;9:18.

21. Suravajhala P, Kogelman LJ, Kadarmideen HN. Multi-omic data integration and analysis using systems genomics approaches: methods and applications in animal production, health and welfare. Genet Sel Evol. 2016;48(1):1.

22. Menke A. Gene expression: Biomarker of antidepressant therapy? Int Rev Psychiatry. 2013;25(5):579-591.

23. Peng B, Li H, Peng X-X. Functional metabolomics: from biomarker discovery to metabolome reprogramming. Protein Cell. 2015;6(9): 628-637.

24. Aagaard K, Petrosino J, Keitel W, et al. The Human Microbiome Project strategy for comprehensive sampling of the human microbiome and why it matters. FASEB J. 2013;27(3):1012-1022.

25. Sonner Z, Wilder E, Heikenfeld J, et al. The microfluidics of the eccrine sweat gland, including biomarker partitioning, transport, and biosensing implications. Biomicrofluidics. 2015;9(3):031301.

26. Schmidt HD, Shelton RC, Duman RS. Functional biomarkers of depression: diagnosis, treatment, and pathophysiology. Neuropsychopharm. 2011;36(12):2375-2394.

27. J Brand S, Moller M, H Harvey B. A review of biomarkers in mood and psychotic disorders: a dissection of clinical vs. preclinical correlates. Curr Neuropharmacol. 2015;13(3):324-368. 
28. Lopresti AL, Maker GL, Hood SD, Drummond PD. A review of peripheral biomarkers in major depression: the potential of inflammatory and oxidative stress biomarkers. Prog Neuropsychopharmacol Biol Psychiatry. 2014;48:102-111.

29. Fu CH, Steiner H, Costafreda SG. Predictive neural biomarkers of clinical response in depression: a meta-analysis of functional and structural neuroimaging studies of pharmacological and psychological therapies. Neurobiol Dis. 2013;52:75-83.

30. Mamdani F, Berlim M, Beaulieu M, Labbe A, Merette C, Turecki G. Gene expression biomarkers of response to citalopram treatment in major depressive disorder. Transl Psychiatry. 2011;1(6):e13.

31. Smith RS. The macrophage theory of depression. Med Hypotheses. 1991; 35(4):298-306.

32. Irwin MR, Miller AH. Depressive disorders and immunity: 20 years of progress and discovery. Brain Behav Immun. 2007;21(4):374-383.

33. Maes M, Leonard B, Myint A, Kubera M, Verkerk R. The new "5-HT" hypothesis of depression: cell-mediated immune activation induces indoleamine 2,3-dioxygenase, which leads to lower plasma tryptophan and an increased synthesis of detrimental tryptophan catabolites (TRYCATs), both of which contribute to the onset of depression. Prog Neuropsychopharmacol Biol Psychiatry. 2011;35(3):702-721.

34. Miller AH, Maletic V, Raison CL. Inflammation and its discontents: The role of cytokines in the pathophysiology of major depression. Biol Psychiatry. 2009;65(9):732-741.

35. Miller AH, Raison CL. The role of inflammation in depression: from evolutionary imperative to modern treatment target. Nat Rev Immun. 2016; 16(1):22-34.

36. Raison CL, Capuron L, Miller AH. Cytokines sing the blues: inflammation and the pathogenesis of depression. Trends Immun. 2006;27(1): 24-31.

37. Raison CL, Felger JC, Miller AH. Inflammation and treatment resistance in major depression: The perfect storm. Psychiatr Times. 2013;30(9).

38. Dowlati Y, Herrmann N, Swardfager W, et al. A meta-analysis of cytokines in major depression. Biol Psychiatry. 2010;67(5):446-457.

39. Eyre HA, Air T, Pradhan A, et al. A meta-analysis of chemokines in major depression. Prog Neuropsychopharmacol Biol Psychiatry. 2016;68:1-8.

40. Haapakoski R, Mathieu J, Ebmeier KP, Alenius H, Kivimäki M. Cumulative meta-analysis of interleukins 6 and $1 \beta$, tumour necrosis factor $\alpha$ and C-reactive protein in patients with major depressive disorder. Brain Behav Immun. 2015;49:206-215.

41. Howren MB, Lamkin DM, Suls J. Associations of depression with C-reactive protein, IL-1, and IL-6: a meta-analysis. Psychosom Med. 2009; 71(2):171-186.

42. Liu Y, Ho RC-M, Mak A. Interleukin (IL)-6, tumour necrosis factor alpha (TNF- $\alpha$ ) and soluble interleukin-2 receptors (sIL-2R) are elevated in patients with major depressive disorder: a meta-analysis and metaregression. $J$ Affect Disord. 2012;139(3):230-239.

43. Strawbridge R, Arnone D, Danese A, Papadopoulos A, Herane Vives A, Cleare AJ. Inflammation and clinical response to treatment in depression: A meta-analysis. Eur Neuropsychopharmacol. 2015;25(10): $1532-1543$.

44. Farooq RK, Asghar K, Kanwal S, Zulqernain A. Role of inflammatory cytokines in depression: Focus on interleukin-1 $\beta$ (Review). Biomed Rep. 2017;6(1):15-20.

45. Cattaneo A, Ferrari C, Uher R, et al. Absolute measurements of macrophage migration inhibitory factor and interleukin-1- $\beta$ mRNA levels accurately predict treatment response in depressed patients. Int J Neuropsychopharmacol. 2016;19(10):pyw045.

46. Baune B, Smith E, Reppermund S, et al. Inflammatory biomarkers predict depressive, but not anxiety symptoms during aging: the prospective Sydney memory and aging study. Psychoneuroendocrinol. 2012;37(9): $1521-1530$.

47. Fornaro M, Rocchi G, Escelsior A, Contini P, Martino M. Might different cytokine trends in depressed patients receiving duloxetine indicate differential biological backgrounds. J Affect Disord. 2013;145(3): 300-307.
48. Hernandez ME, Mendieta D, Martinez-Fong D, et al. Variations in circulating cytokine levels during 52 week course of treatment with SSRI for major depressive disorder. Eur Neuropsychopharmacol. 2008;18(12): 917-924.

49. Hannestad J, DellaGioia N, Bloch M. The effect of antidepressant medication treatment on serum levels of inflammatory cytokines: a meta-analysis. Neuropsychopharmacology. 2011;36(12):2452-2459.

50. Hiles SA, Attia J, Baker AL. Changes in interleukin-6, C-reactive protein and interleukin-10 in people with depression following antidepressant treatment: A meta-analysis. Brain Behav Immun. Presented at: 17th Annual Meeting of the PsychoNeuroImmunology Research Society PsychoNeuroImmunology: Crossing Disciplines to Combat Disease. 2012;24:S44.

51. Harley J, Luty S, Carter J, Mulder R, Joyce P. Elevated C-reactive protein in depression: A predictor of good long-term outcome with antidepressants and poor outcome with psychotherapy. JPsychopharmacol. 2010;24(4):625-626.

52. Uher R, Tansey KE, Dew T, et al. An inflammatory biomarker as a differential predictor of outcome of depression treatment with escitalopram and nortriptyline. Am J Psychiatry. 2014;171(2):1278-1286.

53. Chang HH, Lee IH, Gean PW, et al. Treatment response and cognitive impairment in major depression: Association with C-reactive protein. Brain Behav Immun. 2012;26(1):90-95.

54. Raison CL, Rutherford RE, Woolwine BJ, et al. A randomized controlled trial of the tumor necrosis factor antagonist infliximab for treatment-resistant depression: the role of baseline inflammatory biomarkers. JAMA Psychiatry. 2013;70(1):31-41.

55. Krishnadas R, Cavanagh J. Depression: an inflammatory illness? J Neurol Neurosurg Psychiatry. 2012;83(5):495-502.

56. Raison CL, Miller AH. Is depression an inflammatory disorder? Curr Psychiatry Rep. 2011;13(6):467-475.

57. Simon N, McNamara K, Chow C, et al. A detailed examination of cytokine abnormalities in Major Depressive Disorder. Eur Neuropsychopharmacol. 2008;18(3):230-233.

58. Dahl J, Ormstad H, Aass HC, et al. The plasma levels of various cytokines are increased during ongoing depression and are reduced to normal levels after recovery. Psychoneuroendocrinol. 2014;45:77-86.

59. Stelzhammer V, Haenisch F, Chan MK, et al. Proteomic changes in serum of first onset, antidepressant drug-naïve major depression patients. Int J Neuropsychopharmacol. 2014;17(10):1599-1608.

60. Liu Y, HO RCM, Mak A. The role of interleukin (IL)-17 in anxiety and depression of patients with rheumatoid arthritis. Int J Rheum Dis. 2012;15(2):183-187.

61. Diniz BS, Sibille E, Ding Y, et al. Plasma biosignature and brain pathology related to persistent cognitive impairment in late-life depression. Mol Psychiatry. 2015;20(5):594-601.

62. Janelidze S, Ventorp F, Erhardt S, et al. Altered chemokine levels in the cerebrospinal fluid and plasma of suicide attempters. Psychoneuroendocrinol. 2013;38(6):853-862.

63. Powell TR, Schalkwyk LC, Heffernan AL, et al. Tumor necrosis factor and its targets in the inflammatory cytokine pathway are identified as putative transcriptomic biomarkers for escitalopram response. Eur Neuropsychopharmacol. 2013;23(9):1105-1114.

64. Wong M, Dong C, Maestre-Mesa J, Licinio J. Polymorphisms in inflammation-related genes are associated with susceptibility to major depression and antidepressant response. Mol Psychiatry. 2008;13(8): $800-812$.

65. Kling MA, Alesci S, Csako G, et al. Sustained low-grade pro-inflammatory state in unmedicated, remitted women with major depressive disorder as evidenced by elevated serum levels of the acute phase proteins $C$-reactive protein and serum amyloid A. Biol Psychiatry. 2007;62(4):309-313.

66. Schaefer M, Sarkar S, Schwarz M, Friebe A. Soluble intracellular adhesion molecule-1 in patients with unipolar or bipolar affective disorders: results from a pilot trial. Neuropsychobiol. 2016;74(1):8-14.

67. Dimopoulos N, Piperi C, Salonicioti A, et al. Elevation of plasma concentration of adhesion molecules in late-life depression. Int J Geriatr Psychiatry. 2006;21(10):965-971. 
68. Bocchio-Chiavetto L, Bagnardi V, Zanardini R, et al. Serum and plasma BDNF levels in major depression: a replication study and meta-analyses. World J Biol Psychiatry. 2010;11(6):763-773.

69. Brunoni AR, Lopes M, Fregni F. A systematic review and meta-analysis of clinical studies on major depression and BDNF levels: implications for the role of neuroplasticity in depression. Int J Neuropsychopharmacol. 2008;11(8):1169-1180.

70. Molendijk M, Spinhoven P, Polak M, Bus B, Penninx B, Elzinga B. Serum BDNF concentrations as peripheral manifestations of depression: evidence from a systematic review and meta-analyses on 179 associations. Mol Psychiatry. 2014;19(7):791-800.

71. Sen S, Duman R, Sanacora G. Serum brain-derived neurotrophic factor, depression, and antidepressant medications: meta-analyses and implications. Biol Psychiatry. 2008;64(6):527-532.

72. Zhou L, Xiong J, Lim Y, et al. Upregulation of blood proBDNF and its receptors in major depression. J Affect Disord. 2013;150(3):776-784.

73. Chen Y-W, Lin P-Y, Tu K-Y, Cheng Y-S, Wu C-K, Tseng P-T. Significantly lower nerve growth factor levels in patients with major depressive disorder than in healthy subjects: a meta-analysis and systematic review. Neuropsychiatr Dis Treat. 2014;11:925-933.

74. Lin PY, Tseng PT. Decreased glial cell line-derived neurotrophic factor levels in patients with depression: a meta-analytic study. J Psychiatr Res. 2015;63:20-27.

75. Warner-Schmidt JL, Duman RS. VEGF as a potential target for therapeutic intervention in depression. Curr Op Pharmacol. 2008;8(1):14-19.

76. Carvalho AF, Köhler CA, McIntyre RS, et al. Peripheral vascular endothelial growth factor as a novel depression biomarker: a metaanalysis. Psychoneuroendocrinol. 2015;62:18-26.

77. Tseng P-T, Cheng Y-S, Chen Y-W, Wu C-K, Lin P-Y. Increased levels of vascular endothelial growth factor in patients with major depressive disorder: A meta-analysis. Eur Neuropsychopharmacol. 2015;25(10): 1622-1630.

78. Carvalho L, Torre J, Papadopoulos A, et al. Lack of clinical therapeutic benefit of antidepressants is associated overall activation of the inflammatory system. J Affect Disord. 2013;148(1):136-140.

79. Clark-Raymond A, Meresh E, Hoppensteadt D, et al. Vascular endothelial growth factor: Potential predictor of treatment response in major depression. World J Biol Psychiatry. 2015:1-11.

80. Isung J, Mobarrez F, Nordström P, Åsberg M, Jokinen J. Low plasma vascular endothelial growth factor (VEGF) associated with completed suicide. World J Biol Psychiatry. 2012;13(6):468-473.

81. Buttenschøn HN, Foldager L, Elfving B, Poulsen PH, Uher R, Mors O. Neurotrophic factors in depression in response to treatment. J Affect Disord. 2015;183:287-294.

82. Szczęsny E, Ślusarczyk J, Głombik K, et al. Possible contribution of IGF-1 to depressive disorder. Pharmacol Rep. 2013;65(6):1622-1631.

83. Tu KY, Wu MK, Chen YW, et al. Significantly higher peripheral insulin-like growth factor-1 levels in patients with major depressive disorder or bipolar disorder than in healthy controls: a meta-analysis and review under Guideline of PRISMA. Med. 2016;95(4):e2411.

84. Wu CK, Tseng PT, Chen YW, Tu KY, Lin PY. Significantly higher peripheral fibroblast growth factor-2 levels in patients with major depressive disorder: A preliminary meta-analysis under MOOSE guidelines. Med. 2016;95(33):e4563.

85. He S, Zhang T, Hong B, et al. Decreased serum fibroblast growth factor-2 levels in pre-and post-treatment patients with major depressive disorder. Neurosci Lett. 2014;579:168-172.

86. Dwivedi Y, Rizavi HS, Conley RR, Roberts RC, Tamminga CA, Pandey GN. Altered gene expression of brain-derived neurotrophic factor and receptor tyrosine kinase B in postmortem brain of suicide subjects. Arch Gen Psychiatry. 2003;60(8):804-815.

87. Srikanthan K, Feyh A, Visweshwar H, Shapiro JI, Sodhi K. Systematic review of metabolic syndrome biomarkers: A panel for early detection, management, and risk stratification in the West Virginian population. Int J Med Sci. 2016;13(1):25.

88. Lu X-Y. The leptin hypothesis of depression: a potential link between mood disorders and obesity? Curr Op Pharmacol. 2007;7(6):648-652.
89. Wittekind DA, Kluge M. Ghrelin in psychiatric disorders-A review. Psychoneuroendocrinol. 2015;52:176-194.

90. Kan C, Silva N, Golden SH, et al. A systematic review and metaanalysis of the association between depression and insulin resistance. Diabetes Care. 2013;36(2):480-489.

91. Liu X, Li J, Zheng P, et al. Plasma lipidomics reveals potential lipid markers of major depressive disorder. Anal Bioanal Chem. 2016; 408(23):6497-6507.

92. Lustman PJ, Anderson RJ, Freedland KE, De Groot M, Carney RM, Clouse RE. Depression and poor glycemic control: a meta-analytic review of the literature. Diabetes Care. 2000;23(7):934-942.

93. Maes M. Evidence for an immune response in major depression: a review and hypothesis. Prog NeuroPsychopharmacol Biol Psychiatry. 1995;19(1):11-38.

94. Zheng H, Zheng P, Zhao L, et al. Predictive diagnosis of major depression using NMR-based metabolomics and least-squares support vector machine. Clinica Chimica Acta. 2017;464:223-227.

95. Xia Q, Wang G, Wang H, Xie Z, Fang Y, Li Y. Study of metabolism of glucose and lipid in patients of first-episode depressioon. J Clin Psychiatry. 2009;19:241-243.

96. Kaufman J, DeLorenzo C, Choudhury S, Parsey RV. The 5-HT 1A receptor in major depressive disorder. Eur Neuropsychopharmacology. 2016;26(3):397-410.

97. Jacobsen JP, Krystal AD, Krishnan KRR, Caron MG. Adjunctive 5-Hydroxytryptophan slow-release for treatment-resistant depression: clinical and preclinical rationale. Trends Pharmacol Sci. 2016; 37(11):933-944.

98. Salamone JD, Correa M, Yohn S, Cruz LL, San Miguel N, Alatorre L. The pharmacology of effort-related choice behavior: Dopamine, depression, and individual differences. Behav Processes. 2016;127: 3-17.

99. Coplan JD, Gopinath S, Abdallah CG, Berry BR. A neurobiological hypothesis of treatment-resistant depression-mechanisms for selective serotonin reuptake inhibitor non-efficacy. Front Behav Neurosci. 2014;8:189.

100. Popa D, Cerdan J, Repérant C, et al. A longitudinal study of 5-HT outflow during chronic fluoxetine treatment using a new technique of chronic microdialysis in a highly emotional mouse strain. Eur $J$ Pharmacol. 2010;628(1):83-90.

101. Atake K, Yoshimura R, Hori H, et al. Duloxetine, a selective noradrenaline reuptake inhibitor, increased plasma levels of 3-methoxy4-hydroxyphenylglycol but not homovanillic acid in patients with major depressive disorder. Clin Psychopharmacol Neurosci. 2014; 12(1):37-40.

102. Ueda N, Yoshimura R, Shinkai K, Nakamura J. Plasma levels of catecholamine metabolites predict the response to sulpiride or fluvoxamine in major depression. Pharmacopsychiatry. 2002;35(05):175-181.

103. Yamana M, Atake K, Katsuki A, Hori H, Yoshimura R. Blood biological markers for prediction of escitalopram response in patients with major depressive disorder: preliminary study. J Depress Anxiety. 2016;5:222.

104. Parker KJ, Schatzberg AF, Lyons DM. Neuroendocrine aspects of hypercortisolism in major depression. Horm Behav. 2003;43(1): 60-66.

105. Stetler C, Miller GE. Depression and hypothalamic-pituitary-adrenal activation: a quantitative summary of four decades of research. Psychosom Med. 2011;73(2):114-126.

106. Herane Vives A, De Angel V, Papadopoulos A, et al. The relationship between cortisol, stress and psychiatric illness: New insights using hair analysis. J Psychiatr Res. 2015;70:38-49.

107. Fischer S, Strawbridge R, Vives AH, Cleare AJ. Cortisol as a predictor of psychological therapy response in depressive disorders: systematic review and meta-analysis. Br J Psychiatry. 2017;210(2): 105-109.

108. Anacker C, Zunszain PA, Carvalho LA, Pariante CM. The glucocorticoid receptor: pivot of depression and of antidepressant treatment? Psychoneuroendocrinology. 2011;36(3):415-425. 
109. Markopoulou K, Papadopoulos A, Juruena MF, Poon L, Pariante CM, Cleare AJ. The ratio of cortisol/DHEA in treatment resistant depression. Psychoneuroendocrinol. 2009;34(1):19-26.

110. Joffe RT, Pearce EN, Hennessey JV, Ryan JJ, Stern RA. Subclinical hypothyroidism, mood, and cognition in older adults: a review. Int $J$ Geriatr Psychiatry. 2013;28(2):111-118.

111. Duval F, Mokrani MC, Erb A, et al. Chronobiological hypothalamicpituitary-thyroid axis status and antidepressant outcome in major depression. Psychoneuroendocrinol. 2015;59:71-80.

112. Marsden W. Synaptic plasticity in depression: molecular, cellular and functional correlates. Prog Neuropsychopharmacol Biol Psychiatry. 2013;43:168-184.

113. Duman RS, Voleti B. Signaling pathways underlying the pathophysiology and treatment of depression: novel mechanisms for rapid-acting agents. Trends Neurosci. 2012;35(1):47-56.

114. Ripke S, Wray NR, Lewis CM, et al. A mega-analysis of genome-wide association studies for major depressive disorder. Mol Psychiatry. 2013;18(4):497-511

115. Mullins N, Power R, Fisher H, et al. Polygenic interactions with environmental adversity in the aetiology of major depressive disorder. Psychol Med. 2016;46(04):759-770.

116. Lewis S. Neurological disorders: telomeres and depression. Nat Rev Neurosci. 2014;15(10):632.

117. Lindqvist D, Epel ES, Mellon SH, et al. Psychiatric disorders and leukocyte telomere length: underlying mechanisms linking mental illness with cellular aging. Neurosci Biobehav Rev. 2015;55:333-364.

118. McCall WV. A rest-activity biomarker to predict response to SSRIs in major depressive disorder. J Psychiatr Res. 2015;64:19-22.

119. Schuch FB, Deslandes AC, Stubbs B, Gosmann NP, da Silva CTB, de Almeida Fleck MP. Neurobiological effects of exercise on major depressive disorder: a systematic review. Neurosci Biobehav Rev. 2016; 61:1-11.

120. Foster JA, Neufeld K-AM. Gut-brain axis: how the microbiome influences anxiety and depression. Trends Neurosci. 2013;36(5):305-312.

121. Quattrocki E, Baird A, Yurgelun-Todd D. Biological aspects of the link between smoking and depression. Harv Rev Psychiatry. 2000; $8(3): 99-110$

122. Maes M, Kubera M, Obuchowiczwa E, Goehler L, Brzeszcz J. Depression's multiple comorbidities explained by (neuro)inflammatory and oxidative and nitrosative stress pathways. Neuro Endocrinol Lett. 2011; 32(1):7-24.

123. Miller G, Rohleder N, Cole SW. Chronic interpersonal stress predicts activation of pro- and anti-inflammatory signaling pathways six months later. Psychosom Med. 2009;71(1):57.

124. Steptoe A, Hamer M, Chida Y. The effects of acute psychological stress on circulating inflammatory factors in humans: a review and meta-analysis. Brain Behav Immun. 2007;21(7):901-912.

125. Danese A, Moffitt TE, Harrington H, et al. Adverse childhood experiences and adult risk factors for age-related disease: depression, inflammation, and clustering of metabolic risk markers. Arch Pediatr Adolesc Med. 2009;163(12):1135-1143.

126. Danese A, Pariante CM, Caspi A, Taylor A, Poulton R. Childhood maltreatment predicts adult inflammation in a life-course study. Proc Natl Acad Sci U S A. 2007;104(4):1319-1324.

127. Danese A, Caspi A, Williams B, et al. Biological embedding of stress through inflammation processes in childhood. Mol Psychiatry. 2011;16(3):244-246.

128. Suzuki A, Poon L, Kumari V, Cleare AJ. Fear biases in emotional face processing following childhood trauma as a marker of resilience and vulnerability to depression. Child Maltreat. 2015;20(4):240-250.

129. Strawbridge R, Young AH. HPA axis and cognitive dysregulation in mood disorders. In: McIntyre RS, Cha DS, editors. Cognitive Impairment in Major Depressive Disorder: Clinical Relevance, Biological Substrates, and Treatment Opportunities. Cambridge: Cambridge University Press; 2016:179-193.

130. Keller J, Gomez R, Williams G, et al. HPA axis in major depression: cortisol, clinical symptomatology and genetic variation predict cognition. Mol Psychiatry. Epub 2016 Aug 16.
131. Hanson ND, Owens MJ, Nemeroff CB. Depression, antidepressants, and neurogenesis: a critical reappraisal. Neuropsychopharmacol. 2011; 36(13):2589-2602.

132. Chen Y, Baram TZ. Toward understanding how early-life stress reprograms cognitive and emotional brain networks. Neuropsychopharmacol. 2015;41(1):197-206.

133. Porter RJ, Gallagher P, Thompson JM, Young AH. Neurocognitive impairment in drug-free patients with major depressive disorder. Br J Psychiatry. 2003;182:214-220.

134. Gallagher P, Robinson L, Gray J, Young A, Porter R. Neurocognitive function following remission in major depressive disorder: potential objective marker of response? Aust N Z J Psychiatry. 2007;41(1): 54-61.

135. Pittenger C, Duman RS. Stress, depression, and neuroplasticity: a convergence of mechanisms. Neuropsychopharmacol. 2008;33(1): $88-109$.

136. Bäckman L, Nyberg L, Lindenberger U, Li SC, Farde L. The correlative triad among aging, dopamine, and cognition: current status and future prospects. Neurosci Biobehav Rev. 2006;30(6):791-807.

137. Allison DJ, Ditor DS. The common inflammatory etiology of depression and cognitive impairment: a therapeutic target. J Neuroinflammation. 2014;11:151.

138. Rosenblat JD, Brietzke E, Mansur RB, Maruschak NA, Lee Y, McIntyre RS. Inflammation as a neurobiological substrate of cognitive impairment in bipolar disorder: Evidence, pathophysiology and treatment implications. J Affect Disord. 2015;188:149-159.

139. Krogh J, Benros ME, Jørgensen MB, Vesterager L, Elfving B, Nordentoft $\mathrm{M}$. The association between depressive symptoms, cognitive function, and inflammation in major depression. Brain Behav Immun. 2014;35:70-76.

140. Soares CN, Zitek B. Reproductive hormone sensitivity and risk for depression across the female life cycle: a continuum of vulnerability? J Psychiatry Neurosci. 2008;33(4):331.

141. Hiles SA, Baker AL, de Malmanche T, Attia J. A meta-analysis of differences in IL- 6 and IL-10 between people with and without depression: exploring the causes of heterogeneity. Brain Behav Immun. 2012; 26(7):1180-1188.

142. Fontana L, Eagon JC, Trujillo ME, Scherer PE, Klein S. Visceral fat adipokine secretion is associated with systemic inflammation in obese humans. Diabetes. 2007;56(4):1010-1013.

143. Divani AA, Luo X, Datta YH, Flaherty JD, Panoskaltsis-Mortari A. Effect of oral and vaginal hormonal contraceptives on inflammatory blood biomarkers. Mediators Inflamm. 2015;2015:379501.

144. Ramsey JM, Cooper JD, Penninx BW, Bahn S. Variation in serum biomarkers with sex and female hormonal status: implications for clinical tests. Sci Rep. 2016;6:26947.

145. Eyre H, Lavretsky H, Kartika J, Qassim A, Baune B. Modulatory effects of antidepressant classes on the innate and adaptive immune system in depression. Pharmacopsychiatry. 2016;49(3):85-96.

146. Hiles SA, Baker AL, de Malmanche T, Attia J. Interleukin-6, C-reactive protein and interleukin-10 after antidepressant treatment in people with depression: a meta-analysis. Psychol Med. 2012;42(10): 2015-2026.

147. Janssen DG, Caniato RN, Verster JC, Baune BT. A psychoneuroimmunological review on cytokines involved in antidepressant treatment response. Hum Psychopharmacol. 2010;25(3):201-215.

148. Artigas F. Serotonin receptors involved in antidepressant effects. Pharmacol Ther. 2013;137(1):119-131.

149. Lee B-H, Kim Y-K. The roles of BDNF in the pathophysiology of major depression and in antidepressant treatment. Psychiatry Investig. 2010;7(4):231-235.

150. Hashimoto K. Inflammatory biomarkers as differential predictors of antidepressant response. Int J Mol Sci. 2015;16(4):7796-7801.

151. Goldberg D. The heterogeneity of "major depression". World Psychiatry. 2011;10(3):226-228.

152. Arnow BA, Blasey C, Williams LM, et al. Depression subtypes in predicting antidepressant response: a report from the iSPOT-D trial. Am J Psychiatry. 2015;172(8):743-750. 
153. Kunugi H, Hori H, Ogawa S. Biochemical markers subtyping major depressive disorder. Psychiatry Clin Neurosci. 2015;69(10): 597-608.

154. Baune B, Stuart M, Gilmour A, et al. The relationship between subtypes of depression and cardiovascular disease: a systematic review of biological models. Transl Psychiatry. 2012;2(3):e92.

155. Vogelzangs N, Duivis HE, Beekman AT, et al. Association of depressive disorders, depression characteristics and antidepressant medication with inflammation. Transl Psychiatry. 2012;2:e79.

156. Lamers F, Vogelzangs N, Merikangas K, De Jonge P, Beekman A, Penninx B. Evidence for a differential role of HPA-axis function, inflammation and metabolic syndrome in melancholic versus atypical depression. Mol Psychiatry. 2013;18(6):692-699.

157. Penninx BW, Milaneschi Y, Lamers F, Vogelzangs N. Understanding the somatic consequences of depression: biological mechanisms and the role of depression symptom profile. BMC Med. 2013;11(1):1.

158. Capuron L, Su S, Miller AH, et al. Depressive Symptoms and Metabolic Syndrome: Is Inflammation the Underlying Link? Biol Psychiatry. 2008;64(10):896-900.

159. Dantzer R, O'Connor JC, Freund GG, Johnson RW, Kelley KW. From inflammation to sickness and depression: when the immune system subjugates the brain. Nat Rev Neurosci. 2008;9(1):46-56.

160. Maes M, Berk M, Goehler L, et al. Depression and sickness behavior are Janus-faced responses to shared inflammatory pathways. BMC Med 2012;10:66.

161. Merikangas KR, Jin R, He J-P, et al. Prevalence and correlates of bipolar spectrum disorder in the world mental health survey initiative. Arch Gen Psychiatry. 2011;68(3):241-251.

162. Hirschfeld RM, Lewis L, Vornik LA. Perceptions and impact of bipolar disorder: how far have we really come? Results of the national depressive and manic-depressive association 2000 survey of individuals with bipolar disorder. J Clin Psychiatry. 2003;64(2):161-174.

163. Young AH, MacPherson H. Detection of bipolar disorder. $\mathrm{Br} J$ Psychiatry. 2011;199(1):3-4.

164. Vöhringer PA, Perlis RH. Discriminating between bipolar disorder and major depressive disorder. Psychiatr Clin North Am. 2016; 39(1):1-10.

165. Becking K, Spijker AT, Hoencamp E, Penninx BW, Schoevers RA, Boschloo L. Disturbances in hypothalamic-pituitary-adrenal axis and immunological activity differentiating between unipolar and bipolar depressive episodes. PLoS One. 2015;10(7):e0133898.

166. Huang TL, Lin FC. High-sensitivity C-reactive protein levels in patients with major depressive disorder and bipolar mania. Prog NeuroPsychopharmacol Biol Psychiatry. 2007;31(2):370-372.

167. Angst J, Gamma A, Endrass J. Risk factors for the bipolar and depression spectra. Acta Psychiatr Scand. 2003;418:15-19.

168. Fekadu A, Wooderson S, Donaldson C, et al. A multidimensional tool to quantify treatment resistance in depression: the Maudsley staging method. J Clin Psychiatry. 2009;70(2):177.

169. Papakostas G, Shelton R, Kinrys G, et al. Assessment of a multi-assay, serum-based biological diagnostic test for major depressive disorder: a pilot and replication study. Mol Psychiatry. 2013;18(3):332-339.

170. Fan J, Han F, Liu H. Challenges of big data analysis. Natl Sci Rev. 2014;1(2):293-314

171. Li L, Jiang H, Qiu Y, Ching WK, Vassiliadis VS. Discovery of metabolite biomarkers: flux analysis and reaction-reaction network approach. BMC Syst Biol. 2013;7(Suppl 2):S13.

172. Patel MJ, Khalaf A, Aizenstein HJ. Studying depression using imaging and machine learning methods. NeuroImage Clin. 2016;10:115-123.

173. Lanquillon S, Krieg JC, Bening-Abu-Shach U, Vedder H. Cytokine production and treatment response in major depressive disorder. Neuropsychopharmacol. 2000;22(4):370-379.

174. Lindqvist D, Janelidze S, Erhardt S, Träskman-Bendz L, Engström G, Brundin L. CSF biomarkers in suicide attempters-a principal component analysis. Acta Psychiatr Scand. 2011;124(1):52-61.

175. Hidalgo-Mazzei D, Murru A, Reinares M, Vieta E, Colom F. Big data in mental health: a challenging fragmented future. World Psychiatry. 2016;15(2):186-187.
176. Consortium C-DGotPG. Identification of risk loci with shared effects on five major psychiatric disorders: a genome-wide analysis. Lancet. 2013;381(9875):1371-1379.

177. Dipnall JF, Pasco JA, Berk M, et al. Fusing data mining, machine learning and traditional statistics to detect biomarkers associated with depression. PLoS One. 2016;11(2):e0148195.

178. Köhler O, Benros ME, Nordentoft M, et al. Effect of anti-inflammatory treatment on depression, depressive symptoms, and adverse effects: a systematic review and meta-analysis of randomized clinical trials. JAMA Psychiatry. 2014;71(12):1381-1391.

179. Wolkowitz OM, Reus VI, Chan T, et al. Antiglucocorticoid treatment of depression: double-blind ketoconazole. Biol Psychiatry. 1999;45(8): 1070-1074.

180. McAllister-Williams RH, Anderson IM, Finkelmeyer A, et al. Antidepressant augmentation with metyrapone for treatment-resistant depression (the ADD study): a double-blind, randomised, placebocontrolled trial. Lancet Psychiatry. 2016;3(2):117-127.

181. Gallagher P, Young AH. Mifepristone (RU-486) treatment for depression and psychosis: A review of the therapeutic implications. Neuropsychiatr Dis Treat. 2006;2(1):33-42.

182. Otte C, Hinkelmann K, Moritz S, et al. Modulation of the mineralocorticoid receptor as add-on treatment in depression: a randomized, double-blind, placebo-controlled proof-of-concept study. J Psychiatr Res. 2010;44(6):339-346.

183. Ozbolt LB, Nemeroff CB. HPA axis modulation in the treatment of mood disorders. Psychiatr Disord. 2013;51:1147-1154.

184. Walker AK, Budac DP, Bisulco S, et al. NMDA receptor blockade by ketamine abrogates lipopolysaccharide-induced depressive-like behavior in C57BL/6J mice. Neuropsychopharmacol. 2013;38(9): 1609-1616.

185. Lespérance F, Frasure-Smith N, St-André E, Turecki G, Lespérance P, Wisniewski SR. The efficacy of omega-3 supplementation for major depression: a randomized controlled trial. J Clin Psychiatry. 2010; 72(8):1054-1062.

186. Kim S, Bae K, Kim J, et al. The use of statins for the treatment of depression in patients with acute coronary syndrome. Transl Psychiatry. 2015;5(8):e620.

187. Shishehbor MH, Brennan M-L, Aviles RJ, et al. Statins promote potent systemic antioxidant effects through specific inflammatory pathways. Circulation. 2003;108(4):426-431.

188. Mercier A, Auger-Aubin I, Lebeau J-P, et al. Evidence of prescription of antidepressants for non-psychiatric conditions in primary care: an analysis of guidelines and systematic reviews. BMC Family Practice. 2013;14(1):55.

189. Freland L, Beaulieu J-M. Inhibition of GSK3 by lithium, from single molecules to signaling networks. Front Mol Neurosci. 2012;5:14.

190. Horowitz MA, Zunszain PA. Neuroimmune and neuroendocrine abnormalities in depression: two sides of the same coin. Ann N Y Acad Sci. 2015;1351(1):68-79.

191. Juruena MF, Cleare AJ. Overlap between atypical depression, seasonal affective disorder and chronic fatigue syndrome. Rev Bras Psiquiatr. 2007;29:S19-S26.

192. Castrén E, Kojima M. Brain-derived neurotrophic factor in mood disorders and antidepressant treatments. Neurobiol Dis. 2017; 97(Pt B):119-126.

193. Pan A, Keum N, Okereke OI, et al. Bidirectional association between depression and metabolic syndrome a systematic review and metaanalysis of epidemiological studies. Diabetes Care. 2012;35(5): 1171-1180.

194. Carvalho AF, Rocha DQ, McIntyre RS, et al. Adipokines as emerging depression biomarkers: a systematic review and meta-analysis. J Psychiatric Res. 2014;59:28-37.

195. Wise T, Cleare AJ, Herane A, Young AH, Arnone D. Diagnostic and therapeutic utility of neuroimaging in depression: an overview. Neuropsychiatr Dis Treat. 2014;10:1509-1522.

196. Tamatam A, Khanum F, Bawa AS. Genetic biomarkers of depression. Indian J Hum Genet. 2012;18(1):20. 
197. Yoshimura R, Nakamura J, Shinkai K, Ueda N. Clinical response to antidepressant treatment and 3-methoxy-4-hydroxyphenylglycol levels: mini review. Prog Neuropsychopharmacol Biol Psychiatry. 2004; 28(4):611-616.

198. Pierscionek T, Adekunte O, Watson S, Ferrier N, Alabi A. Role of corticosteroids in the antidepressant response. ChronoPhys Ther. 2014; 4:87-98.

199. Hage MP, Azar ST. The link between thyroid function and depression. J Thyroid Res. 2012;2012:590648.
200. Dunn EC, Brown RC, Dai Y, et al. Genetic determinants of depression: recent findings and future directions. Harv Rev Psychiatry. 2015; 23(1):1.

201. Yang C-C, Hsu Y-L. A review of accelerometry-based wearable motion detectors for physical activity monitoring. Sensors. 2010;10(8): $7772-7788$.

\section{Publish your work in this journal}

Neuropsychiatric Disease and Treatment is an international, peerreviewed journal of clinical therapeutics and pharmacology focusing on concise rapid reporting of clinical or pre-clinical studies on a range of neuropsychiatric and neurological disorders. This journal is indexed on PubMed Central, the 'PsycINFO' database and CAS, and is the official journal of The International Neuropsychiatric Association (INA). The manuscript management system is completely online and includes a very quick and fair peer-review system, which is all easy to use. Visit http://www.dovepress.com/testimonials.php to read real quotes from published authors.

\footnotetext{
Submit your manuscript here: http://www.dovepress.com/neuropsychiatric-disease-and-treatment-journal
} 